\title{
Evaluation Parameter of Excessive Daytime Sleepiness for Obstructive Sleep Apnea Patients
}

\author{
Sang Hoo Park, MD, Woo Yong Bae, MD, PhD, Sangjun Kim, MD, Young Gun Kim, MD \\ Department of Otorhinolaryngology-Head and Neck Surgery, Dong-A University College of Medicine, Busan, Korea
}

Received: November 12, 2021

Revised: December 6, 2021

Accepted: December 21, 2021

Correspondence

Woo Yong Bae, MD, PhD

Department of Otorhinolaryngology-

Head and Neck Surgery,

Dong-A University College of Medicine,

26 Daesingongwon-ro, Seo-gu,

Busan 49201, Korea

Tel +82-51-240-5428

Fax +82-51-253-0712

E-mail_doncamel@dau.ac.kr

\section{ORCID}

Sang Hoo Park

https://orcid.org/0000-0002-5994-2428

Woo Yong Bae

https://orcid.org/0000-0001-5578-0225

Sangjun Kim

https://orcid.org/0000-0002-6025-6775

Young Gun Kim

https://orcid.org/0000-0002-4874-4311

(c) This is an Open Access article distributed under the terms of the Creative Commons Attribution Non-Commercial License (https://creativecommons.org/licenses/by-nc/4.0) which permits unrestricted non-commercial use, distribution, and reproduction in any medium, provided the original work is properly cited.
Background and Objective Obstructive sleep apnea (OSA) is a sleep-related breathing disorder caused by repetitive obstruction of the upper airway. Repetitive obstruction of the upper airway causes impaired gaseous exchange, resulting hypoxia, hypercapnia, and frequent arousals of sleep architecture. Polysomnography (PSG) is a gold standard for diagnosing OSA. Excessive daytime sleepiness (EDS) is a common accompanying daytime symptoms in OSA patients. Since EDS can cause unexpected events such as traffic accident or poor performance in workplace, it is regarded as a significant public health problem. Therefore, accurate assessment and prediction of this symptom is important. The Epworth Sleepiness Scale (ESS) and multiple sleep latency test are most commonly used to evaluate EDS, but their efficacies are controversial. The purpose of this study is to find the parameter to evaluate and predict the EDS for OSA patients.

Methods We retrospectively reviewed the medical records of 88 OSA patients. Patients were divided into two groups according to the presence of EDS. We analyzed the clinical records, questionnaire scores, and PSG data to find the difference between two groups.

Results ESS was $10.64 \pm 4.28$ in EDS patients and $8.63 \pm 4.86$ in non-EDS patients. ESS showed a statistically significant difference between two groups $(\mathrm{p}=0.044)$. Also, the percentage of 1st stage non-REM sleep in total sleep time (N1\%) was 25.09 \pm 15.24 in EDS patients and $18.97 \pm 10.30$ in non-EDS patients and showed a statistically significant difference between groups $(\mathrm{p}=0.033)$. Patients' weight was $81.59 \pm 20.52$ in EDS patients and 74.14 \pm 12.63 in non-EDS patients and showed a statistically significant difference between groups $(\mathrm{p}=0.046)$.

Conclusions ESS, N1\% and patients' weight were significant parameter which is related with the presence of EDS for OSA patients. These parameters will be useful in evaluating the presence of EDS for OSA patients. Also, in patients diagnosed with sleep disorder with high N1\%, EDS must be accurately evaluated as well.

Sleep Med Res 2021;12(2):110-117

Keywords Obstructive sleep apnea; Polysomnography; Excessive daytime sleepiness; Epworth Sleepiness Scale.

\section{INTRODUCTION}

Obstructive sleep apnea (OSA) is a common condition with a prevalence of 3\%-7\% in adult males and $2 \%-5 \%$ in adult females [1]. It is characterized by repeated obstruction of the upper airways during sleep which causes physiological dissonance. Consequently, the dissonance results in sleep symptoms such as snoring, apnea, dyspnea, and daytime symptoms including excessive daytime sleepiness (EDS) and headache [2]. In addition, repeated obstruction of the upper airways is known to induce arousal during sleep due to apnea and suffocation, which can lead to cardiovascular diseases such as arrhythmia, hypertension, coronary artery disease, and cerebrovascular diseases (such as stroke), and various metabolic diseases [3-5].

EDS is defined as a compulsion to sleep during the major waking episodes of the day [6]. It may fatal consequences such as poor performance at work [7], and car accidents; traffic acci- 
dents due to daytime sleepiness are reported to account for approximately $10 \%$ to $30 \%$ of all traffic accidents [8]. The multiple sleep latency test (MSLT), which is an objective test, and the Epworth Sleep Scale (ESS), which is a subjective test, are the most commonly used EDS evaluation methods. However, MSLT may show varying results depending on the time of the day. In addition, it is limited by cost, time, and space because the test is conducted using electroencephalogram (EEG) [9]. Compared with MSLT, ESS is relatively simple to perform and there are no time and space limitations, but it is subjective and there are still many controversies about the accuracy of the test results.

EDS has been associated with personal, economical, and social challenges; therefore, it is necessary to accurately identify the reason why it is common in OSA patients. In addition, accurate evaluation and prediction of OSA are essential for appropriate treatment.

This study aimed to objectively evaluate and predict EDS in patients with OSA, and to find out whether positive airway pressure (PAP) treatment is useful in treating EDS.

\section{METHODS}

\section{Subjects}

The study targeted patients who visited our hospital sleep clinic with suggestive OSA symptoms and underwent polysomnography (PSG) from January 2019 to December 2019. Patients with an apnea-hypopnea index (AHI) of less than 5 or diagnosed with central or mixed sleep apnea were excluded from the study; finally, a total of 88 patients were included in the study.

\section{Study Design}

A retrospective study was conducted through chart analysis of 88 subjects. Institutional Ethics Committee approval was granted by our institution's IRB (DAUHIRB-21-103).

\section{Definition of EDS}

Before performing PSG, we asked whether the patients felt sleepy during daytime activities such as at the workplace or while driving. Based on patients' answers, they were divided into EDS and non-EDS groups.

\section{Clinical Data}

The patients' age, sex, and history were studied. History included the history of smoking, hypertension, diabetes, myocardial infarction, and cerebrovascular disease. Systolic, diastolic blood pressure, blood glucose, total cholesterol, high, low-density cholesterol, and triglyceride levels were also checked.

\section{Questionnaire}

All the patients completed questionnaires related to various psychiatric symptoms including anxiety and depression; this in- cluded Patient Health Questionnaire-15 [10], Beck Anxiety Inventory [11], Beck's Depression Inventory [11], Maudsley Obsessive-Compulsive Inventory [12], Social Avoidance, and Distress Scale [13], and Fear of Negative Evaluation [13]. Also, patients completed questionnaires about how they subjectively felt about their sleep quality; this included Pittsburgh Sleep Quality Index [14], Global Sleep Assessment Questionnaire [15], ESS [9], and Stanford Sleepiness Scale [16].

\section{PSG Data}

After PSG was performed, total sleep time, sleep efficiency, the ratio of sleep stages (N1\%, N2\%, N3\%), and REM sleep ratio (REM\%) were analyzed. Arousal index, apnea index, hypopnea index, AHI, respiratory disturbance index (RDI), lowest oxygen saturation during sleep, and duration were recorded simultaneously. Sleep efficiency was defined as the ratio of the total sleep time to the total recorded time, and the arousal index was defined as the number of awakenings divided by the total sleep time.

Apnea is defined as the cessation of airflow for at least $10 \mathrm{sec}-$ onds. Hypopnea is defined as a recognizable transient reduction of breathing for 10 seconds or longer, or a decrease of greater than $50 \%$ in the amplitude of a validated measure of breathing, or a reduction in amplitude of less than $50 \%$ associated with oxygen desaturation of $4 \%$ or more. Respiratory effort-related arousal is defined as an event characterized by increasing respiratory effort for 10 seconds or longer leading to an arousal from sleep but does not fulfill the criteria for hypopnea or apnea. AHI is defined as the average number of apnea and hypopnea episodes per hour. The RDI is defined as the average number of respiratory disturbances (obstructive apneas, hypopneas, and respiratory event related arousals) per hour.

\section{Obesity}

The height, weight, body mass index (BMI), neck and waist circumference which could be confirmed on the chart, were identified as obesity-related values.

\section{Compliance of PAP}

Among the patients with EDS, the use of PAP device were analyzed. The percentage of days using PAP device based on 90 days (PAP\%) and the percentage of days using PAP device for more than 4 hours (Time4\%) were analyzed.

\section{Statistical Analysis}

SPSS 24.0 (IBM Corp., Armonk, NY, USA) was used as the analysis program and all statistical significance was set to 0.05 . Patients were classified into with and without EDS groups, and a statistical difference test was performed for the previously mentioned items between the two groups. T-test was performed to analyze the relationship between the results of PAP device use and symptom improvement. 


\section{RESULTS}

\section{Clinical Data}

There were 68 males (77.3\%) and 20 females (22.7\%) with an average age of $51.36 \pm 13.28$ years. Forty-six patients (52.3\%) had EDS and 42 patients (47.7\%) had no EDS. Among the patients with EDS, 37 patients were treated with PAP, among them 17 patients showed improvement while 20 patients did not.

There were no differences in age and gender ratio between pa- tients with and without EDS. Also, there were no differences in the history of smoking, hypertension, diabetes, myocardial infarction, and cerebrovascular disease between the two groups. Systolic, diastolic blood pressure, blood glucose, total cholesterol, high, low-density cholesterol, and triglyceride levels showed no statistical difference between the two groups. Also, there was no statistical difference between mild-to-moderate and severe sleep apnea based on the severity evaluation results as shown by AHI and $\mathrm{RDI}$ values (Table 1 ).

Table 1. Demographic data of enrolled patients diagnosed as OSA

\begin{tabular}{|c|c|c|c|c|}
\hline & EDS patients $(n=46)$ & Non-EDS patients $(\mathrm{n}=42)$ & Total $(\mathrm{n}=88)$ & $\mathrm{p}$-value \\
\hline Age (years) & $50.02 \pm 13.32$ & $52.62 \pm 13.35$ & $51.36 \pm 13.28$ & 0.364 \\
\hline Sex & & & & 0.459 \\
\hline Male & $37(80.4)$ & $31(73.8)$ & $68(77.3)$ & \\
\hline Female & $9(19.6)$ & $11(26.2)$ & $20(22.7)$ & \\
\hline Smoking & & & & 0.759 \\
\hline Yes & $19(41.3)$ & $16(38.1)$ & $35(39.8)$ & \\
\hline No & $27(58.7)$ & $26(61.9)$ & $53(60.2)$ & \\
\hline Hypertension & & & & 0.098 \\
\hline Yes & $14(30.4)$ & $20(47.6)$ & $34(38.6)$ & \\
\hline No & $32(69.6)$ & $22(52.4)$ & $54(61.4)$ & \\
\hline Systolic blood pressure & $140.37 \pm 16.78$ & $138.20 \pm 19.46$ & $139.15 \pm 17.97$ & 0.619 \\
\hline Diastolic blood pressure & $82.29 \pm 13.55$ & $79.26 \pm 12.26$ & $80.76 \pm 12.83$ & 0.330 \\
\hline Total cholesterole & $193.31 \pm 30.73$ & $184.21 \pm 37.55$ & $188.70 \pm 33.82$ & 0.336 \\
\hline HDL-cholesterole & $52.24 \pm 11.33$ & $56.22 \pm 14.13$ & $53.58 \pm 12.91$ & 0.265 \\
\hline LDL-cholesterole & $118.04 \pm 24.29$ & $115.68 \pm 29.53$ & $116.80 \pm 26.23$ & 0.758 \\
\hline Triglyceride & $160.41 \pm 83.89$ & $155.91 \pm 80.29$ & $158.55 \pm 80.76$ & 0.846 \\
\hline Diabetes mellitus & & & & 0.509 \\
\hline Yes & $42(91.3)$ & $36(85.7)$ & $78(88.6)$ & \\
\hline No & $4(8.7)$ & $6(14.3)$ & $10(11.4)$ & \\
\hline Glucose & $106.57 \pm 16.05$ & $117.35 \pm 47.02$ & $111.88 \pm 33.61$ & 0.261 \\
\hline Myocardial infarction & & & & 0.419 \\
\hline Yes & $2(4.3)$ & $4(9.5)$ & $6(6.8)$ & \\
\hline No & $44(95.7)$ & $38(90.5)$ & $82(93.2)$ & \\
\hline Cerebral vascular accident & & & & 0.188 \\
\hline Yes & $1(2.2)$ & $4(9.5)$ & $5(5.7)$ & \\
\hline No & $45(97.8)$ & $38(90.5)$ & 83 (94.3) & \\
\hline OSA groups & & & & 0.361 \\
\hline Mild to moderate $(5<\mathrm{AHI} \leq 30)$ & $23(48.0)$ & $16(40.0)$ & $39(44.3)$ & \\
\hline Severe $(30 \leq \mathrm{AHI})$ & $25(52.0)$ & $24(60.0)$ & $49(55.7)$ & \\
\hline OSA groups & & & & 0.788 \\
\hline Mild to moderate $(5<\mathrm{RDI} \leq 30)$ & $21(43.8)$ & $17(42.5)$ & $38(43.2)$ & \\
\hline Severe $(30 \leq \mathrm{RDI})$ & $27(56.2)$ & $23(57.7)$ & $50(56.8)$ & \\
\hline
\end{tabular}

Data are presented as mean \pm standard deviation.

EDS, excessive daytime sleepiness; HDL, high-density cholesterol; LDL, low-density cholesterol; OSA, obstructive sleep apnea; AHI, apneahypopnea index; RDI, respiratory disturbance index. 


\section{Questionnaire}

Results of Patient Health Questionnaire-15, Beck Anxiety Inventory, Beck's Depression Inventory, Maudsley Obsessive-Compulsive Inventory, Social Avoidance and Distress Scale, and Fear of Negative Evaluation showed no statistical difference between the two groups (Table 2).

Among the questionnaires about how they subjectively felt about their sleep, ESS was $10.64 \pm 4.28$ in the EDS group and $8.63 \pm 4.86$ in the non-EDS group; ESS showed a statistically significant difference between the two groups $(\mathrm{p}=0.044)$. On the other hand, the other questionnaires showed no statistical differences between the groups (Table 3).

\section{PSG Data}

Among the PSG items related to the sleep stage, N1\% which is the ratio of stage 1 non-REM sleep among the proportion of sleep stages was $25.09 \pm 15.24$ in the EDS group and $18.97 \pm 10.30$ in the non-EDS group showing a statistically significant difference between the two groups ( $\mathrm{p}=0.033$ ). Total sleep time, sleep efficiency, N2\%, N3\%, and REM\% showed no statistically significant differences (Table 4).

Arousal index which reflects sleep fragmentation, apnea index, hypopnea index, and AHI that determines the severity of OSA showed no statistically significant difference. RDI, lowest oxygen saturation during sleep, and duration also showed no statistically significant differences (Table 5).

\section{Obesity}

Among obesity-related values, weight was $81.59 \pm 20.52$ in the EDS group and $74.14 \pm 12.63$ in the non-EDS group. Weight showed a statistically significant difference between the two groups ( $p=0.046)$. Other obesity-related values showed no statistical difference between the two groups (Table 6).

Table 2. The difference in depression-related questionnaire scores between EDS and non-EDS patients

\begin{tabular}{lcccc}
\hline & EDS patients $(\mathrm{n}=46)$ & Non-EDS patients $(\mathrm{n}=42)$ & Total $(\mathrm{n}=88)$ & $\mathrm{p}$-value \\
\hline PHQ-15 & $6.27 \pm 4.32$ & $5.38 \pm 4.50$ & $5.82 \pm 4.38$ & 0.351 \\
BAI & $8.11 \pm 7.63$ & $7.83 \pm 9.25$ & $7.92 \pm 8.38$ & 0.878 \\
BDI & $11.61 \pm 6.88$ & $13.07 \pm 7.94$ & $12.22 \pm 7.43$ & 0.365 \\
MOCI & $24.61 \pm 16.04$ & $23.76 \pm 17.51$ & $23.93 \pm 16.77$ & 0.814 \\
SADS & $75.10 \pm 13.00$ & $71.46 \pm 20.33$ & $73.41 \pm 16.74$ & 0.422 \\
FNE & $29.53 \pm 5.84$ & $32.73 \pm 9.85$ & $31.02 \pm 8.04$ & 0.139 \\
\hline
\end{tabular}

Data are presented as mean \pm standard deviation.

EDS, excessive daytime sleepiness; PHQ-15, Patient Health Questionnaire 15; BAI, Beck's Anxiety Inventory; BDI, Beck's Depression Inventory; MOCI, Maudsley Obsessive-Compulsive Inventory; SADS, Social Anxiety Distress Scale; FNE, Fear of Negative Evaluation.

Table 3. The difference in sleep quality-related questionnaire scores between EDS and non-EDS patients

\begin{tabular}{lcccc}
\hline & EDS patients $(\mathrm{n}=46)$ & Non-EDS patients $(\mathrm{n}=42)$ & Total $(\mathrm{n}=88)$ & $\mathrm{p}$-value \\
\hline PSQI & $16.09 \pm 5.47$ & $13.22 \pm 7.38$ & $14.73 \pm 6.41$ & 0.166 \\
GSAQ & $11.45 \pm 5.48$ & $8.65 \pm 3.95$ & $10.20 \pm 4.96$ & 0.083 \\
ESS & $10.64 \pm 4.28$ & $8.63 \pm 4.86$ & $9.66 \pm 4.63$ & 0.044 \\
SSS & $2.58 \pm 1.06$ & $2.46 \pm 0.98$ & $2.53 \pm 1.01$ & 0.604 \\
\hline
\end{tabular}

Data are presented as mean \pm standard deviation.

EDS, excessive daytime sleepiness; PSQI, Pittsburgh Sleep Quality Index; GSAQ, Global Sleep Assessment Questionnaire; ESS, Epworth Sleepiness Scale; SSS, Stanford Sleepiness Scale.

Table 4. The difference in polysomnographic data related to sleep stage between EDS and non-EDS patients

\begin{tabular}{lcccc}
\hline & EDS patients $(\mathrm{n}=46)$ & Non-EDS patients $(\mathrm{n}=42)$ & Total $(\mathrm{n}=88)$ & $\mathrm{p}$-value \\
\hline TST & $335.46 \pm 64.28$ & $315.98 \pm 40.96$ & $326.86 \pm 55.07$ & 0.100 \\
S-efficiency & $87.53 \pm 8.78$ & $83.26 \pm 12.42$ & $85.63 \pm 10.70$ & 0.071 \\
N1\% & $25.09 \pm 15.24$ & $18.97 \pm 10.30$ & $22.25 \pm 13.36$ & 0.033 \\
N2\% & $55.57 \pm 11.05$ & $58.32 \pm 8.85$ & $56.72 \pm 10.14$ & 0.207 \\
N3\% & $7.97 \pm 7.43$ & $11.60 \pm 15.62$ & $9.68 \pm 12.00$ & 0.162 \\
REM\% & $11.64 \pm 5.91$ & $13.19 \pm 4.93$ & $12.46 \pm 5.53$ & 0.191 \\
\hline
\end{tabular}

Data are presented as mean \pm standard deviation.

EDS, excessive daytime sleepiness; TST, total sleep time; S-efficiency, sleep efficiency. 
Table 5. The difference in polysomnographic data related to the severity of OSA and sleep fragmentation between EDS and non-EDS patients

\begin{tabular}{lcccc}
\hline & EDS patients $(\mathrm{n}=46)$ & Non-EDS patients $(\mathrm{n}=42)$ & Total $(\mathrm{n}=88)$ & $\mathrm{p}$-value \\
\hline AI & $18.72 \pm 25.90$ & $13.74 \pm 20.19$ & $16.47 \pm 23.27$ & 0.325 \\
HI & $12.98 \pm 11.13$ & $15.76 \pm 13.00$ & $14.23 \pm 12.00$ & 0.286 \\
AHI & $31.32 \pm 27.77$ & $28.67 \pm 24.22$ & $30.11 \pm 25.89$ & 0.639 \\
RDI & $32.05 \pm 28.51$ & $28.70 \pm 25.88$ & $30.51 \pm 27.04$ & 0.570 \\
Low O2 & $79.24 \pm 11.68$ & $81.85 \pm 15.37$ & $80.38 \pm 13.48$ & 0.372 \\
Duration & $8.96 \pm 13.57$ & $20.93 \pm 50.11$ & $14.40 \pm 35.66$ & 0.123 \\
Arousal index & $30.92 \pm 22.02$ & $29.49 \pm 19.00$ & $30.28 \pm 20.43$ & 0.749 \\
\hline
\end{tabular}

Data are presented as mean \pm standard deviation.

EDS, excessive daytime sleepiness; AI, apnea index; HI, hypopnea index; AHI, apnea-hypopnea index; RDI, respiratory disturbance index; OSA, obstructive sleep apnea.

Table 6. The difference in obesity-related factors between EDS and non-EDS patients

\begin{tabular}{lcccc}
\hline & EDS patients $(\mathrm{n}=46)$ & Non-EDS patients $(\mathrm{n}=42)$ & Total $(\mathrm{n}=88)$ & $\mathrm{p}$-value \\
\hline Height & $170.11 \pm 7.62$ & $168.80 \pm 7.93$ & $169.44 \pm 7.72$ & 0.433 \\
Weight & $81.59 \pm 20.52$ & $74.14 \pm 12.63$ & $77.84 \pm 17.44$ & 0.046 \\
BMI & $27.91 \pm 5.63$ & $25.91 \pm 3.86$ & $26.91 \pm 4.92$ & 0.058 \\
Neck & $40.90 \pm 4.17$ & $40.39 \pm 7.63$ & $40.64 \pm 5.97$ & 0.711 \\
Waist & $99.33 \pm 15.09$ & $93.80 \pm 11.51$ & $96.66 \pm 13.67$ & 0.091 \\
\hline
\end{tabular}

Data are presented as mean \pm standard deviation.

EDS, excessive daytime sleepiness; BMI, body mass index.

Table 7. The difference in PAP usage

\begin{tabular}{lccc}
\hline & $\begin{array}{c}\text { Improved patients } \\
(\mathrm{n}=17)\end{array}$ & $\begin{array}{c}\text { Non-improved patients } \\
(\mathrm{n}=20)\end{array}$ & p-value \\
\hline PAP\% & $94.41 \pm 5.16$ & $75.87 \pm 26.40$ & 0.006 \\
Time4\% & $88.26 \pm 9.65$ & $64.34 \pm 28.35$ & 0.002 \\
\hline
\end{tabular}

Data are presented as mean \pm standard deviation. PAP\%, percentage of days using PAP device based on 90 days; Time4\%, percentage of days using PAP device for more than 4 hours.

\section{PAP Device Compliance}

Thirty-seven patients with EDS used a PAP device among them, 17 patients, showed improvement while 20 patients did not show improvement. PAP\% and Time4\% were compared and analyzed using a t-test. PAP\% was $94.41 \pm 5.16$ in the improved group and $75.87 \pm 26.40$ in the non-improved group, showing a statistically significant difference $(\mathrm{p}=0.006)$. Time $4 \%$ was $88.26 \pm 9.65$ in the improved group and $64.34 \pm 28.35$ in the non-improved group, showing a statistically significant difference $(p=0.002)($ Table 7$)$.

\section{DISCUSSION}

OSA results from repeated partial or total obstruction of the upper airways during sleep which consequently causes a repeated decrease in blood oxygen saturation, arousal from sleep, and subsequent sleep fragmentation. It is known that sleep fragmen- tation disrupts the structure of normal sleep, so, the quality of sleep deteriorates, and patients complain of EDS [17]. EDS is difficult to clearly define and evaluate, because the meaning is not precisely defined, so the meaning varies among individual patients [18].

The most commonly used test tools for EDS are subjective test ESS and objective test MSLT. ESS is a questionnaire with a total of 24 points that present 8 situations that can make patients sleepy during the day and has a score of 0 to 3 depending on the frequency of sleeping episodes for each situation. In general, a score of 0 to 10 is interpreted as normal, a score of 11 to 12 as mild EDS, a score of 13 to 15 as moderate EDS, and a score of 16 to 24 as severe EDS. A study by Cho et al. [19] reported that KESS, which is a Korean version of ESS translated according to the Korean cultural and social situation is a reliable and valid tool for the evaluation of patients with EDS. Although ESS is a simple questionnaire and used by many hospitals, studies reported that results are different depending on not only the patient's social and cultural situation, but also anxiety, depression, and gender, making it hard to accurately evaluate EDS [20,21]. The authors who developed the questionnaire also reported that ESS results cannot fully reflect EDS because ESS reflects the subjective sleep state experienced by the patient [22].

MSLT is a more objective test than ESS because it measures patients' sleep latency. Patients lie still in the examination room and the time it takes to fall asleep is measured on the EEG. Sleep latency is measured 4 times at regular intervals on the same day 
and the average value is displayed as the final result [23].

However, MSLT is also affected by limitations in accurately assessing EDS because it is highly affected by the patient's sleep environment, quality, and quantity of sleep during the night before [24].

Several studies have been conducted to identify the factors associated with EDS. Since sleep disturbance is not the only factor associated with EDS, various factors must be considered; mood factors such as depression and metabolic disorders like obesity and diabetes are known to be associated with EDS. Also, studies have reported that EDS appears to be more prevalent in old age because of increasing medical issues, so age can also be an associated contributing factor [25].

We performed various questionnaires to evaluate EDS. The Patient Health Questionnaire-15 consists of 15 items including headache, dizziness, and chest pain, and the degree of patients' subjective pain over the past 4 weeks is indicated on a scale of 0 to 2 [10]. Beck Anxiety Inventory is a questionnaire consisting of a total of 21 items including the cognitive, emotional, and physical domains of anxiety, and the degree of anxiety can be expressed from 0 to 3 [11]. Maudsley Obsessive-Compulsive Inventory is a questionnaire consisting of 30 situations that can occur if you have obsessive-compulsive disorder. This questionnaire is counted as two points for yes and 1 point for no [12]. Social Avoidance and Distress Scale consists of 28 items related to social adaptation, and is marked on a scale of 1 to 5 , with higher the scores indicating a greater degree of anxiety about or avoiding social adaptation. Fear of Negative Evaluation consists of 12 items about interpersonal relationships and is rated on a scale of 1 to 5 , with higher scores indicating greater fear of interpersonal relationships [13].

Pittsburgh Sleep Quality Index consists of 19 questions evaluating 7 items: subjective sleep quality, sleep latency, sleep duration, usual sleep efficiency, sleep disturbance, use of sleeping pills, and daytime dysfunction. Higher scores indicate lower sleep quality [14]. Global Sleep Assessment Questionnaire consists of 11 questions about the quality of patients' sleep during the past 4 weeks [15]. Stanford Sleepiness Scale is a questionnaire about how sleepy patients are currently on a scale of 1 to 7 [16].

In our study, ESS was the only questionnaire that showed a statistically significant difference between EDS and the non-EDS groups. Other questionnaires showed no statistical difference between the two groups. Therefore, ESS is thought to be meaningful in evaluating EDS as confirmed in several studies.

Depression and anxiety are known to be major factors exacerbating OSA, and depression is common in patients diagnosed with OSA [26]. 70\% of patients diagnosed with depression have difficulties falling asleep or maintaining sleep, and resulting insomnia and fatigue can lead to EDS [27].

In this study, six questionnaires that could indirectly diagnose various psychiatric symptoms including anxiety and depression were conducted along with PSG but the results of all the six ques- tionnaires were not statistically significant. These results might suggest that the severity of depression and anxiety does not necessarily correlate with the severity of OSA, and with the incidence of EDS.

Since sleep fragmentation can cause EDS, we predicted that there would be items correlated with EDS among the items of PSG and analyzed them. While studies are reporting that the degree of snoring, sleep efficiency, and arousal index are related to EDS [28], studies by Lee et al. [6] reported that there were no items that showed a significant difference. Oksenberg et al. [29] reported that among patients diagnosed with OSA, the more severe the severity of sleep apnea, the more severe the complaints of EDS were, and items reflecting this such as apnea index, hypopnea index, and AHI were statistically significantly different. Therefore, we predicted that the arousal index which reflects sleep fragmentation, apnea index, hypopnea index, and AHI that determines the severity of OSA, and items such as lowest oxygen saturation and duration could predict EDS, but none of them could be confirmed statistically and this was consistent with the results of Lee et al's study [6]. PSG is used as a standard test for determining the OSA patient's sleep structure of, but it can only determine the sleep structure of for the day of the test.

Stepnowsky et al. [30] performed PSG on 1091 patients for 3 consecutive days, and found differences in the $\mathrm{AHI}$ in about $10 \%$ of patients. Considering this limitation, since one-day PSG test cannot identify all the patients' sleep structure and sleep fragmentation, it was not possible to identify items correlated with EDS.

Our study results confirmed that the higher the N1\% which is the ratio of stage 1 non-REM sleep among the proportion of sleep stages in the PSG, the more the patient complained of EDS.

Stage 1 non-REM sleep is an intermediate stage between awakening and sleep, and can be observed during the process of falling asleep or waking up during sleep, and accounts for $2 \%-5 \%$ of the total sleep time in normal sleep. In the present study, N1\% was $25.09 \pm 15.24$ in the EDS group and $18.97 \pm 10.30$ in the non-EDS group, showing a statistically significant difference between groups. N2\% and N3\% did not show statistically significant differences. This suggests that if the 1st stage of non-REM sleep, which is the shallowest stage of the sleep process, takes more part, the overall quality of sleep decreases, and the patient complains EDS more. Since the total sleep time did not show a statistically significant difference, it can be concluded that how deeply and well a patient's sleep is in the entire sleep process is more important in evaluating EDS than how much a patients sleep. In addition, it can be predicted that EDS may appear in patients diagnosed with sleep disorders, which accounts for a high percentage of N1\% among patients diagnosed with sleep disturbance. REM\%, which represents the proportion of REM sleep out of the total sleep time, showed no statistically significant difference.

Obesity is a well-known cause of OSA and has been consistently reported to be associated with $\operatorname{EDS}[31,32]$. The incidence 
of obesity is increasing, and studies have reported that obese patients complain of EDS about twice as much as non-obese patients [25]. Metabolic disorders and chronic inflammation occurring in obese patients may be associated with these symptoms. Adipose tissue is the largest endocrine organ that produces adipokines which cause inflammation related to various metabolic processes in the body [33]. Vgontzas et al. [34] reported that due to this abnormal metabolic process, obese patients become hyper-aroused at night, resulting in sleep fragmentation and EDS. Also, in obese patients, there are circadian changes in REM sleep occur, making it easier to fall asleep during the day than at night. In this study, to check the correlation between obesity and EDS, the patient's height, weight, BMI index, neck and waist circumference were checked. The average weight was $81.59 \pm 20.52$ in the EDS group and $74.14 \pm 12.63$ in the non-EDS group showing a statistically significant difference between the two groups.

Patients with EDS consume high amounts of caffeine to relieve symptoms, but unfortunately, caffeine makes the symptoms worse because it increases sleep latency and decreases total sleep time and REM sleep duration [35]. Modafinil is recommended as a treatment for EDS, if modafinil is not effective enough, additional psychostimulants such as methylphenidate or amphetamine can be helpful [36]. As a non-drug treatment, there is a report that PAP device, which is the main treatment for OSA, is also an effective treatment for EDS [37]. If oxygen is continuously supplied through the PAP device, decrease in oxygen saturation and arousal during sleep decrease and no sleep fragmentation occur, allowing the patient to maintain a normal sleep structure, thereby improving EDS.

In this study, 37 patients with EDS who used a PAP device for treatment were divided into two groups: 17 patients with improved symptoms and 20 patients with no improvement in symptoms. PAP\% and Time $4 \%$ were compared and analyzed using a t-test between the two groups. A statistically significant difference was confirmed, so it can be interpreted that EDS can be improved if the PAP device is used for a long enough time.

There have been several studies reporting that there was no improvement in EDS despite the use of a PAP device. Pépin et al. [38] reported that there were no significant differences in sex, BMI, or estimated sleep duration between PAP device users with or without improvement in EDS. There is also a report that patients with EDS use PAP devices less than patients without EDS, so we must consider the possibility that patients with EDS use fewer PAP devices because they subjectively feel that EDS does not improve [39].

Since the total number of subjects was only 88 , additional research is required including a larger number of subjects in the future. Since EDS is not only a personal problem, but also an economic and social problem, it must be accurately predicted and treated in the process of diagnosing patients with OSA. For an accurate diagnosis, EDS should be evaluated using PSG as well as careful history taking and ESS questionnaire. In addition, it is important to check the items of PSG and explain to patients with high N1\% in advance that EDS may occur and how to prevent it.

\section{Conclusion}

In patients diagnosed with OSA, ESS, N1\%, and patient's weight can help in predict and evaluate EDS. The three can be used as basic data when establishing treatment plans in the future. Also, it is important to predict EDS in patients diagnosed with sleep disturbance with a high percentage of N1\%. In addition, using a PAP device for a long enough time can help treat EDS.

\section{Availability of Data and Material}

The datasets generated or analyzed during the study are available from the corresponding author on reasonable request.

\section{Author Contributions}

Conceptualization: Bae WY. Data curation: Kim S. Formal analysis: Kim YG. Funding acquisition: Bae WY. Investigation: Park SH. Methodology: Park SH. Project administration: Bae WY. Resources: Kim S. Software: Kim YG. Supervision: Kim S. Validation: Park SH. Visualization: Park SH. Writing_original draft: Park SH. Writing_review \& editing: Park SH.

\section{Conflicts of Interest}

The authors have no potential conflicts of interest to disclose.

\section{Funding Statement}

This work was supported by the Dong-A University research fund.

\section{REFERENCES}

1. Punjabi NM. The epidemiology of adult obstructive sleep apnea. Proc Am Thorac Soc 2008;5:136-43.

2. Redline S, Strohl KP. Recognition and consequences of obstructive sleep apnea hypopnea syndrome. Clin Chest Med 1998;19:1-19.

3. Albuquerque FN, Calvin AD, Sert Kuniyoshi FH, Konecny T, LopezJimenez F, Pressman GS, et al. Sleep-disordered breathing and excessive daytime sleepiness in patients with atrial fibrillation. Chest 2012;141: 967-73.

4. Arzt M, Young T, Finn L, Skatrud JB, Bradley TD. Association of sleepdisordered breathing and the occurrence of stroke. Am J Respir Crit Care Med 2005;172:1447-51.

5. Bassetti C, Aldrich MS. Sleep apnea in acute cerebrovascular diseases: final report on 128 patients. Sleep 1999;22:217-23.

6. Lee D, Lee H, Kang B, Kim J, Kim J, Park S. Excessive daytime sleepiness in patients with obstructive sleep apnea. J Korean Sleep Res Soc 2008;5:43-8.

7. Haavisto ML, Porkka-Heiskanen T, Hublin C, Härmä M, Mutanen P, Müller K, et al. Sleep restriction for the duration of a work week impairs multitasking performance. J Sleep Res 2010;19:444-54.

8. Connor J, Norton R, Ameratunga S, Robinson E, Civil I, Dunn R, et al. Driver sleepiness and risk of serious injury to car occupants: population based case control study. BMJ 2002;324:1125.

9. Johns MW. Sensitivity and specificity of the multiple sleep latency test (MSLT), the maintenance of wakefulness test and the epworth sleepiness scale: failure of the MSLT as a gold standard. J Sleep Res 2000;9:511.

10. Kroenke K, Spitzer RL, Williams JB. The PHQ-15: validity of a new measure for evaluating the severity of somatic symptoms. Psychosom Med 2002;64:258-66.

11. Beck AT, Epstein N, Brown G, Steer RA. An inventory for measuring clinical anxiety: psychometric properties. J Consult Clin Psychol 1988; 
56:893-7.

12. Chan DW. The Maudsley Obsessional-Compulsive Inventory: a psychometric investigation on Chinese normal subjects. Behav Res Ther 1990;28:413-20.

13. Heimberg RG, Hope DA, Rapee RM, Bruch MA. The validity of the social avoidance and distress scale and the fear of negative evaluation scale with social phobic patients. Behav Res Ther 1988;26:407-13.

14. Buysse DJ, Reynolds CF 3rd, Monk TH, Berman SR, Kupfer DJ. The Pittsburgh sleep quality index: a new instrument for psychiatric practice and research. Psychiatry Res 1989;28:193-213.

15. Klingman KJ, Jungquist CR, Perlis ML. Questionnaires that screen for multiple sleep disorders. Sleep Med Rev 2017;32:37-44.

16. MacLean AW, Fekken GC, Saskin P, Knowles JB. Psychometric evaluation of the stanford sleepiness scale. J Sleep Res 1992;1:35-9.

17. Chervin RD. The multiple sleep latency test and Epworth sleepiness scale in the assessment of daytime sleepiness. J Sleep Res 2000;9:399401.

18. Johns M. Rethinking the assessment of sleepiness. Sleep Med Rev 1998; 2:3-15.

19. Cho YW, Lee JH, Son HK, Lee SH, Shin C, Johns MW. The reliability and validity of the Korean version of the Epworth sleepiness scale. Sleep Breath 2011;15:377-84.

20. Johns MW. Daytime sleepiness, snoring, and obstructive sleep apnea. The Epworth sleepiness scale. Chest 1993;103:30-6.

21. Shim EJ, Kim JS, Lee JH, Lee H, Hwang SH, Ahn BH, et al. Prevalence and related parameters of daytime sleepiness in patients with habitual snoring. J Korean Neurol Assoc 2007;25:488-93.

22. Slater G, Steier J. Excessive daytime sleepiness in sleep disorders. J Thorac Dis 2012;4:608-16.

23. Han SJ, Joo EY, Cho JW, Hong SB. The usefulness of repeated multiple sleep latency test for the diagnosis of narcolepsy. J Kor Sleep Soc 2008;5: 39-42.

24. Bonnet MH, Arand DL. Impact of motivation on multiple sleep latency test and maintenance of wakefulness test measurements. J Clin Sleep Med 2005;1:386-90.

25. Bixler EO, Vgontzas AN, Lin HM, Calhoun SL, Vela-Bueno A, Kales A. Excessive daytime sleepiness in a general population sample: the role of sleep apnea, age, obesity, diabetes, and depression. J Clin Endocrinol Metab 2005;90:4510-5.
26. Ejaz SM, Khawaja IS, Bhatia S, Hurwitz TD. Obstructive sleep apnea and depression: a review. Innov Clin Neurosci 2011;8:17-25.

27. McCall WV, Blocker JN, D’Agostino R Jr, Kimball J, Boggs N, Lasater $\mathrm{B}$, et al. Insomnia severity is an indicator of suicidal ideation during a depression clinical trial. Sleep Med 2010;11:822-7.

28. Seneviratne U, Puvanendran K. Excessive daytime sleepiness in obstructive sleep apnea: prevalence, severity, and predictors. Sleep Med 2004;5:339-43.

29. Oksenberg A, Arons E, Nasser K, Shneor O, Radwan H, Silverberg DS. Severe obstructive sleep apnea: sleepy versus nonsleepy patients. $L a$ ryngoscope 2010;120:643-8.

30. Stepnowsky CJ Jr, Orr WC, Davidson TM. Nightly variability of sleepdisordered breathing measured over 3 nights. Otolaryngol Head Neck Surg 2004;131:837-43.

31. Mediano O, Barceló A, de la Peña M, Gozal D, Agusti A, Barbé F. Daytime sleepiness and polysomnographic variables in sleep apnoea patients. Rev Port Pneumol 2007;13:896-8.

32. Steier J, Jolley CJ, Seymour J, Roughton M, Polkey MI, Moxham J. Neural respiratory drive in obesity. Thorax 2009;64:719-25.

33. Ouchi N, Parker JL, Lugus JJ, Walsh K. Adipokines in inflammation and metabolic disease. Nat Rev Immunol 2011;11:85-97.

34. Vgontzas AN, Tan TL, Bixler EO, Martin LF, Shubert D, Kales A. Sleep apnea and sleep disruption in obese patients. Arch Intern Med 1994; 154:1705-11.

35. Lasagna L. Dose-related sleep disturbances induced by coffee and caffeine. Clin Pharmacol Ther 1977;21:244.

36. Takenoshita S, Nishino S. Pharmacologic management of excessive daytime sleepiness. Sleep Med Clin 2017;12:461-78.

37. Patel SR, White DP, Malhotra A, Stanchina ML, Ayas NT. Continuous positive airway pressure therapy for treating sleepiness in a diverse population with obstructive sleep apnea: results of a meta-analysis. Arch Intern Med 2003;163:565-71.

38. Pépin JL, Viot-Blanc V, Escourrou P, Racineux JL, Sapene M, Lévy P, et al. Prevalence of residual excessive sleepiness in CPAP-treated sleep apnoea patients: the French multicentre study. Eur Respir J 2009;33:1062-7.

39. Gasa M, Tamisier R, Launois SH, Sapene M, Martin F, Stach B, et al. Residual sleepiness in sleep apnea patients treated by continuous positive airway pressure. J Sleep Res 2013;22:389-97. 